\title{
The different faces of transitional discs
}

\author{
M. de Juan Ovelar ${ }^{1}$, M. Min ${ }^{2}$, C. Dominik ${ }^{2,3}$, C. Thalmann ${ }^{2}$, \\ P. Pinilla ${ }^{4}$, M. Benisty ${ }^{5}$ and T. Birnstiel ${ }^{6}$ \\ ${ }^{1}$ Leiden Observatory, Leiden University, \\ 2300RA Leiden, The Netherlands \\ email: mjovelar@strw.leidenuniv.nl \\ ${ }^{2}$ Astronomical Institute Anton Pannekoek, University of Amsterdam, \\ 1090 GE Amsterdam, The Netherlands \\ ${ }^{3}$ Department of Astrophysics/IMAPP, Radboud University Nijmegen, \\ 6500 GL Nijmegen, The Netherlands \\ ${ }^{4}$ Institut für Theoretische Astrophysik, Universität Heidelberg, \\ Albert-Ueberle-Straße 2, 69120 Heidelberg, Germany \\ ${ }^{5}$ Institut de Planètologie et Astrophysique Grenoble, \\ 414 rue de la Piscine, 38400 st. Martin d'Heres, France \\ ${ }^{6}$ Harvard-Smithsonian Center for Astrophysics, \\ 60 Garden Street, Cambridge, MA 02138, USA
}

\begin{abstract}
Recent imaging observations of transitional discs have revealed discrepancies between the structure observed at different wavelengths. In some targets, the gap measured using sub$\mathrm{mm}$ observations disappears when observed using near infrared polarimetry, suggesting that the empty region is actually filled with small particles of dust (Dong et al., 2012). Assuming the gapped structure observed in transitional discs is caused by the presence of a planet, we try to explain such discrepancies simulating observations of physical models of disc/planet systems with VLT/SPHERE-ZIMPOL, Subaru/HiCIAO, VLT/VISIR and ALMA.
\end{abstract}

Keywords. Transition Discs - Protoplanetary discs - (Stars:) circumstellar matter

\section{Method}

In order to generate realistic images of a transitional disc, we use similar models as those presented in Pinilla et al. (2012a). These combine 2-D hydrodynamical and 1-D dust evolution simulations including coagulation, fragmentation and radial drift, to self consistently reproduce the evolution of the gas and dust present in the disc. We focus on the cases of a disc with a planet of masses $M_{\mathrm{p}}=[1,9,15] \mathrm{M}_{\text {jup }}$. after $3 \mathrm{Myr}$ of evolution. The resulting distribution of dust and gas for these three cases, after $3 \mathrm{Myr}$ of evolution, is input into the Monte-Carlo radiative transfer code MCMax ( Min et al., 2009), to produce theoretical intensity and polarised intensity images of the emitted and scattered flux of the disc. We then simulate realistic observations with VLT/SPHERE, Subaru/HiCIAO and ALMA at wavelengths $\lambda=[0.65,1.6,850] \mu \mathrm{m}$ using specific instrument simulators (SPHERE-ZIMPOL (Thalmann et al. 2008) or convolving the full resolution images with an either measured or simulated point-spread-function (PSF).

\section{Results and Conclusions}

Figure 1 shows the synthetic observations of the disc-planet systems studied.

(a) The trapping and filtering mechanisms triggered by the presence of a $>1$ Mjup mass planet in the disc yield a distinction in the radial distribution of the dust particles 


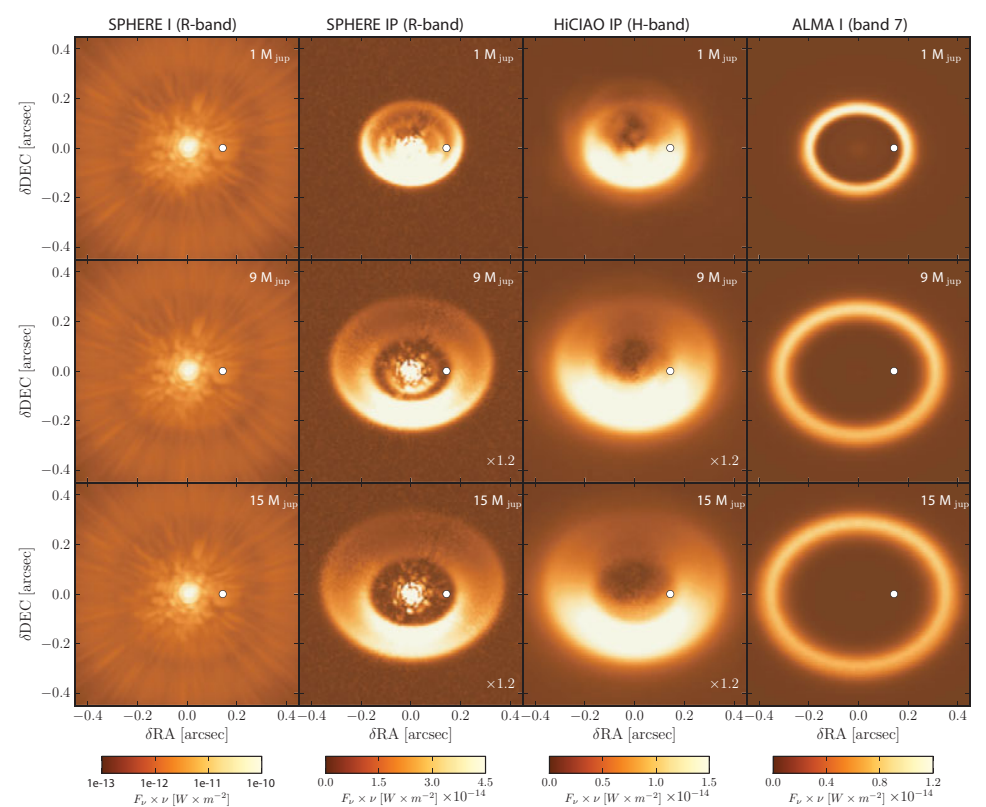

Figure 1. Simulated images of a $3 \mathrm{Myr}$ disc model with a planet orbiting at $20 \mathrm{AU}$ (white dot). Columns show images of intensity (I) and polarised intensity (PI) in the R-band $(0.65 \mu \mathrm{m})$ with SPHERE-ZIMPOL, PI in the H-band $(1.6 \mu \mathrm{m})$ with HiCIAO and intensity with ALMA at $850 \mu \mathrm{m}$. Top, middle and bottom rows show images obtained for planet masses of $M_{p}=[1,9,15] \mathrm{M}_{\mathrm{jup}}$ respectively. The system has an inclination of $i=35^{\circ}$

according to their size. This causes observations at different wavelengths to show different structures. Particles with larger sizes tend to be trapped in the region outside the planet's orbit, while smaller particles are allowed to move to the inner radii. The former show up in sub-mm emission measurements while the latter are detected in polarised scattering flux.

(b) The position of the pressure "trap", the amount of particles trapped and filtered, and the particle size threshold of the filtering process depend strongly on the mass of the planet. This effect makes possible to constrain planet mass and position by combining multi-wavelength observations.

(c) Combination of sub-mm and polarimetric images at visible wavelengths, is the best imaging "strategy" to characterise the main features of this dust grain size distribution. An instrument like SPHERE-ZIMPOL in the R-band $(\lambda=0.65 \mu \mathrm{m})$ is able to differentiate between the three planet mass cases studied in polarised intensity, particularly if the system is inclined.

\section{References}

Dong, R., Rafikov, R., Zhu, Z., et al. 2012, ApJ, 750, 161

Min, M., Dullemond, C. P., Dominik, C., Koter, A. D., \& Hovenier, J. W. 2009, A\&A, 497, 155

Pinilla, P., Benisty, M., \& Birnstiel, T. 2012a, A\&A, 545, A81

Thalmann, C., Schmid, H. M., Boccaletti, A., et al. 2008, SPIE, Vol. 7014 